\title{
Bright Line Detection in COSMO-SkyMed SAR Images of Urban Areas
}

\author{
P.T.B. Brett and R. Guida \\ Surrey Space Centre \\ University of Surrey \\ Guildford, UK \\ p.brett@surrey.ac.uk
}

\begin{abstract}
Bright lines are a characteristic feature of synthetic aperture radar (SAR) amplitude images of urban areas, and are commonly associated with man-made structures. In order to aid in the development of SAR applications using these features, an automated approach to bright line detection is proposed, based on scale-space ridge detection at a single scale, and using a naïve Bayesian classification step to select the ridge points corresponding to bright lines. The effectiveness of the technique is demonstrated by applying it to a COSMO-SkyMed image of L'Aquila, Italy.
\end{abstract}

\section{INTRODUCTION}

The launches of the COSMO-SkyMed constellation and the TerraSAR-X mission have made very high resolution Synthetic Aperture Radar (SAR) data widely available for civilian remote sensing applications, greatly increasing the level of detail visible in SAR images of urban areas.

A characteristic feature of metre-resolution SAR amplitude images of urban areas are bright, narrow lines, which arise from the geometry of man-made structures. For example, bright lines can be caused by direct specular scattering from surfaces normal to the sensor's look vector, such as gabled roofs, or by multiple-reflection scattering from two or more adjacent and mutually perpendicular surfaces, such as a building's wall and an adjacent paved road. Optical models have been developed for the analysis and simulation of building backscatter [1], and these have been inverted and applied to double-reflection lines manually extracted from SAR images in order to estimate parameters such as building height [2], [3], [4].

In order to make it easier to take advantage of bright line features in urban SAR image interpretation, an automated method for identifying points in a SAR image that lie on bright lines was desired. This paper presents a novel approach to bright line extraction based on the detection of ridge points (described in Sec. II) and subsequent classification of detected ridge points in order to remove dim and/or weak lines (Sec. III). It includes the results of applying the method to a SAR amplitude image from COSMO-SkyMed (Sec. IV).

\section{RIDGE POINT DETECTION}

\section{A. Height definition for ridge points}

Detecting bright lines in the SAR image is an application of ridge detection. There are a large number of different definitions of a 'ridge' (an overview of many of the more common ones is provided by [5]) and it is therefore necessary to clarify that the ridge detection method described in this paper is based on the height definition for a ridge. Let $f$ : $\mathbb{R}^{2} \rightarrow \mathbb{R}_{+}$be a SAR amplitude image. Define the negated Hessian matrix of $f, W=-\operatorname{Hess}(f)$, and let $k_{1}, k_{2}$ and $\boldsymbol{\nu}_{1}, \boldsymbol{\nu}_{2}$ be its eigenvalues and eigenvectors, ordered such that $\left|k_{1}\right| \geq\left|k_{2}\right|$. Then a point in the image $\boldsymbol{x}$ is a bright ridge point if:

$$
k_{1}>0 \quad \text { and } \quad \boldsymbol{\nu}_{1}^{T} \nabla f(\boldsymbol{x})=0 .
$$

\section{B. Ridge detection in scale-space}

Based on this height definition of a ridge point, [6] describes a scale-space approach to ridge detection, and a brief summary of this method follows, in order to introduce terminology used later in this paper. The scale-space representation $L: \mathbb{R}^{2} \times$ $\mathbb{R}_{+} \rightarrow \mathbb{R}$ of the image $f$ is defined by $L(\boldsymbol{x} ; t)=g(\boldsymbol{x} ; t) * f$ where the scale parameter $t$ is the variance of the Gaussian kernel $g: \mathbb{R}^{2} \times \mathbb{R}_{+} \rightarrow \mathbb{R}$. The scale-space derivatives are defined by $L_{x^{\alpha} y^{\beta}}(\boldsymbol{x} ; t)=\partial_{x^{\alpha} y^{\beta}} L(\boldsymbol{x} ; t)$, where $(\alpha, \beta)$ denotes the order of differentiation. At any image point $\boldsymbol{x}_{0}$ a local $(p, q)$ coordinate system is defined, aligned with $\boldsymbol{\nu}_{1}, \boldsymbol{\nu}_{2}$, where $W$ is formed from the second-order scale-space derivatives:

$$
W(\boldsymbol{x} ; t)=-\left[\begin{array}{ll}
L_{x x}(\boldsymbol{x} ; t) & L_{x y}(\boldsymbol{x} ; t) \\
L_{x y}(\boldsymbol{x} ; t) & L_{y y}(\boldsymbol{x} ; t)
\end{array}\right]
$$

By analogy to (1), a point $\boldsymbol{x}_{0}$ is bright ridge point of scale $t$ if:

$$
L_{p p}\left(\boldsymbol{x}_{0} ; t\right)<0 \quad \text { and } \quad L_{p}\left(\boldsymbol{x}_{0} ; t\right)=0 .
$$

\section{Adaptation to SAR urban bright line detection}

Finite impulse response (FIR) filter approximations are used for $g$ and the derivative operator $\partial$, and these are provided by the separable discrete scale-space (DSS) formulation proposed by [7]. Using these small, separable filters has the great advantage of allowing the scale-space to be computed quickly and efficiently.

Instead of extracting ridge segments as described in [6], the implementation described in this paper extracts only points. Once the ridge metrics $L_{p p}$ and $L_{p}$ have been computed, bright ridge points are found by first locating zero-crossing points of 
$L_{p}$ by linear interpolation along rows and along columns, and then testing the sign of the corresponding interpolated value of $L_{p p}$.

The most computationally expensive step of the ridge detection algorithm described here is the eigenvector and eigenvalue decomposition of $W$ for each pixel in the scale-space. Fortunately, this is suited for a multithreaded implementation, as is the separable convolution of the image $f$ with the scale and derivative filters. This allows the ridge detection program to take full advantage of modern multicore CPU architectures.

A method is described in [6] for multi-scale ridge detection, whereby a normalised ridge strength metric $\mathcal{R}_{\text {norm }} L$ is used to select the optimal scale for a ridge point. Unfortunately, for a typical SAR image (with size of order $10^{8}$ pixels), prohibitively large amounts of memory are required for the creation of the scale-space and ridge metrics. Instead, the approach presented in this paper is based on carrying out ridge extraction at a single characteristic scale $t_{c}$. The development of an efficient multi-scale approach is a problem currently under study.

\section{RIDGE POINT CLASSIFICATION}

Ridge point detection detects both strong ridges (e.g. double-reflection lines) and weaker ones (e.g. arising from random noise in the image). A classification step allows ridge points that are of interest to be distinguished from those that are not. In this paper, a naïve Bayes classifier [8] is used to classify each detected ridge point $\boldsymbol{x}$. Two mutually-exclusive classes $B$ and $\bar{B}$ are defined ('bright line' and 'not bright line' respectively), with two feature variables.

The first feature variable $R_{1}$ is the linearly-interpolated SAR backscattering amplitude at $\boldsymbol{x}$, i.e. $r_{1}=f(\boldsymbol{x})$. It is modelled by a $\mathcal{G}_{A}^{0}$ distribution, $R_{1} \sim \mathcal{G}_{A}^{0}(\alpha, \gamma, n)$, which has been demonstrated by to be a viable model for highly heterogeneous urban scatterers [9], [10]. The estimated parameters $\hat{\boldsymbol{\theta}}_{1}=$ $\{\hat{\alpha}, \hat{\gamma}, \hat{n}\}$ are derived from the training data using the logmoment method described in [10].

The second feature variable $R_{2}$ is the the $\gamma$-normalised square principle curvature difference $r_{2}=\mathcal{N}_{\gamma-\operatorname{norm}} L(\boldsymbol{x})$ [6], a measure of ridge strength at $\boldsymbol{x}$, where

$$
\begin{aligned}
\mathcal{N}_{\gamma-\operatorname{norm}} L= & t^{4 \gamma}\left(L_{x x}+L_{y y}\right)^{2} \times \\
& \left(\left(L_{x x}-L_{y y}\right)^{2}+4 L_{x y}^{2}\right) .
\end{aligned}
$$

The authors propose to model the distribution of $R_{2}$ by the log-normal distribution $R_{2} \sim \ln N\left(\mu, \sigma^{2}\right)$, and to use the maximum a posteriori (MAP) parameter estimates $\hat{\boldsymbol{\theta}}_{2}=$ $\{\hat{\mu}, \hat{\sigma}\}$ from the training data.

The MAP decision rule is used to determine into which class a ridge point $\boldsymbol{x}$ should be placed:

$$
\ln \frac{p(B)}{p(\bar{B})}+\sum_{i=1}^{2} \ln \frac{p\left(r_{i}(\boldsymbol{x}) \mid B\right)}{p\left(r_{i}(\boldsymbol{x}) \mid \bar{B}\right)} \underset{B}{\gtrless} 0 .
$$

This approach allows both the 'brightness' and the 'sharpness' of a ridge to be taken into account in the classification.

\begin{tabular}{cccccc}
\hline Feature & \multicolumn{2}{c}{$R_{1} \sim \mathcal{G}_{A}^{0}(\alpha, \gamma, n)$} & \multicolumn{2}{c}{$R_{2} \sim \ln N\left(\mu, \sigma^{2}\right)$} \\
\hline Parameter & $\hat{\alpha}$ & $\hat{\gamma}$ & $\hat{n}$ & $\hat{\mu}$ & $\hat{\sigma}$ \\
\hline \hline$B$ & -2.25 & $1.45 \times 10^{5}$ & 4.90 & 15.5 & 3.02 \\
\hline $\bar{B}$ & -1.64 & $1.29 \times 10^{4}$ & 6.72 & 7.24 & 4.40 \\
\hline \hline$p(B)$ & \multicolumn{5}{c}{0.12} \\
\hline
\end{tabular}

TABLE I: Estimated parameters for Bayesian classifier.

\section{RESUlts}

The ridge detection and ridge point removal algorithms were tested by applying them to a single-look SAR amplitude image of L'Aquila, Italy, acquired by the COSMO-SkyMed constellation on 22nd March 2009. The image has an azimuth and range resolution of $0.8 \mathrm{~m}$.

\section{A. Estimation of $t_{c}$}

First, a characteristic scale $t_{c}$ to be used for ridge point detection was determined. A sample set of $N=70$ bright line points $\left\{\boldsymbol{x}_{i}\right\}$ in the SAR image were manually identified, and the corresponding optimal scales

$$
t_{\mathrm{opt}, i}=\underset{t}{\arg \max } \mathcal{N}_{\gamma-\text { norm }} L\left(\boldsymbol{x}_{i}, t\right)
$$

were found. $t_{c}$ was chosen as the mean of $t_{\mathrm{opt}}$, rounded to the nearest $1 / 3$. The value found was $t_{c}=31 / 3$.

\section{B. L'Aquila barracks}

A military barracks in the northwest of L'Aquila was chosen as a target site for training the classifier. The site has two properties that make it suitable:

- Well-separated buildings, reducing the problems of layover and shadow.

- Buildings with relatively simple geometry, providing a variety of well-defined bright lines.

A $256 \times 256$ pixel SAR amplitude image segment containing the barracks is shown in Fig. 1a. The 17510 ridge points detected with $t_{c}=31 / 3$ were classified using a supervised process, as shown in Fig. 1b, and this classification was used to train the Bayesian classifier. The estimated parameters are shown in Table I, and the training data histograms and corresponding estimated probability density functions (PDFs) are shown in Fig. 2.

To verify the classifier, it was used to classify the ridge points of the training image (Fig 1c and Fig. 1d). The Bayesian classifier classified $9.80 \%$ of the ridge points differently to the supervised classification $(7.16 \%$ false alarms and $2.65 \%$ misses). A qualitative assessment of the results suggested that the high false alarm rate was due to the Bayesian classification correctly classifying bright lines that had been omitted from the supervised classification. 


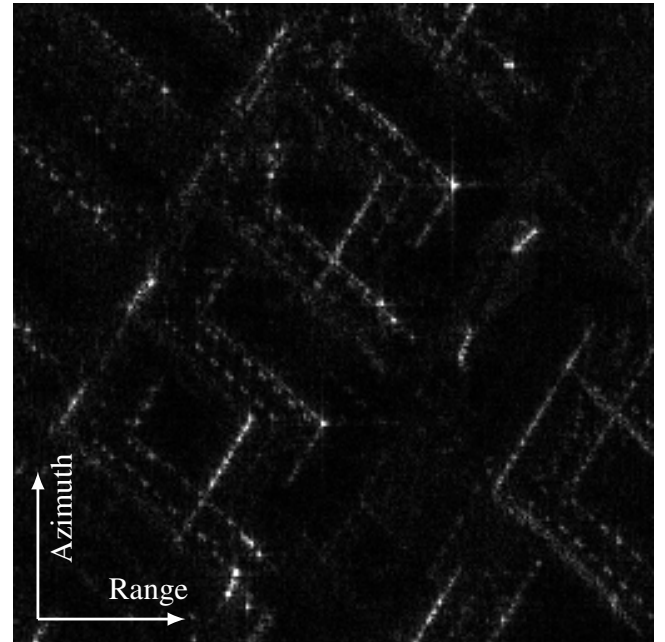

(a) Original image.

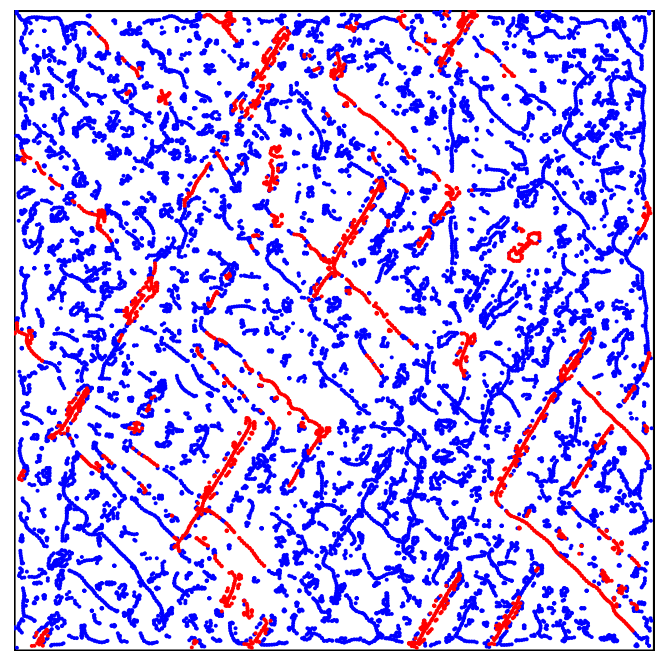

(c) Bayesian classification of detected ridge points $(B$ shown in red, $\bar{B}$ in blue).

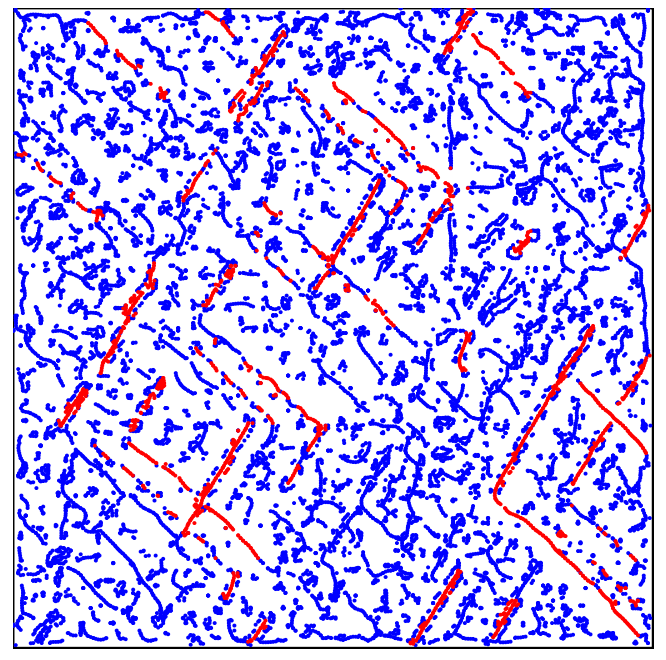

(b) Supervised classification of detected ridge points ( $B$ shown in red, $\bar{B}$ in blue)).

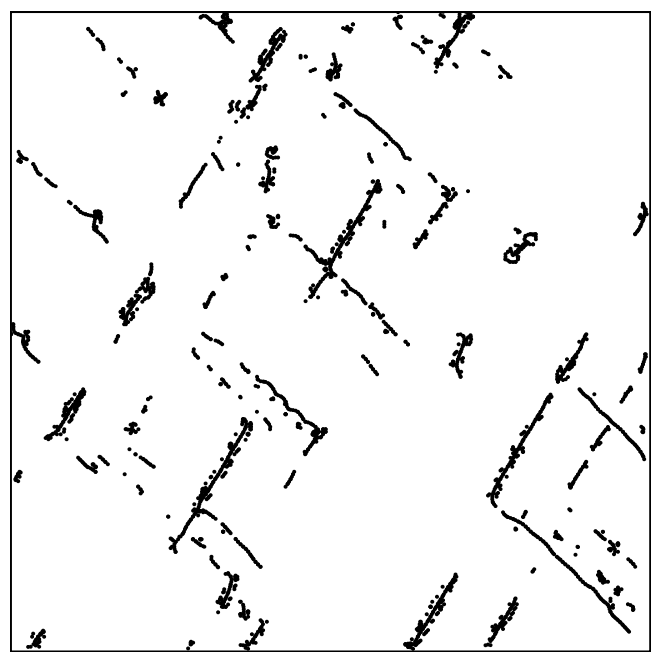

(d) Bayesian classification of detected ridge points ( $B$ only).

Fig. 1: L’Aquila barracks bright line detection results. Processed from COSMO-SkyMed product @ Agenzia Spaziale Italiana 2009. All rights reserved.
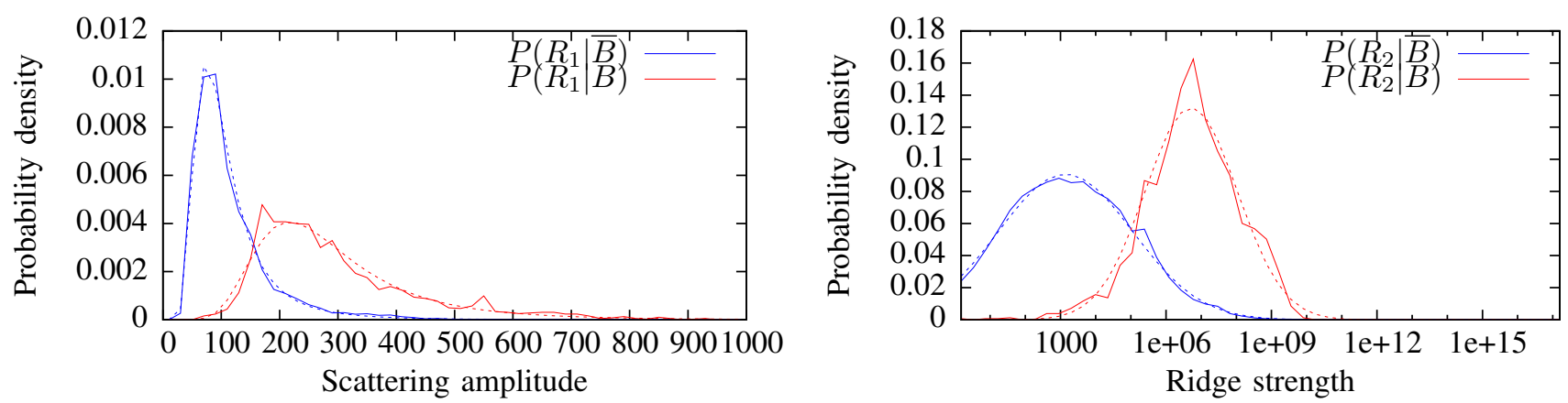

Fig. 2: MAP probability distributions of $R_{1}$ and $R_{2}$ after training. The training data histogram is plotted with lines, and the estimated feature models with dashes. 


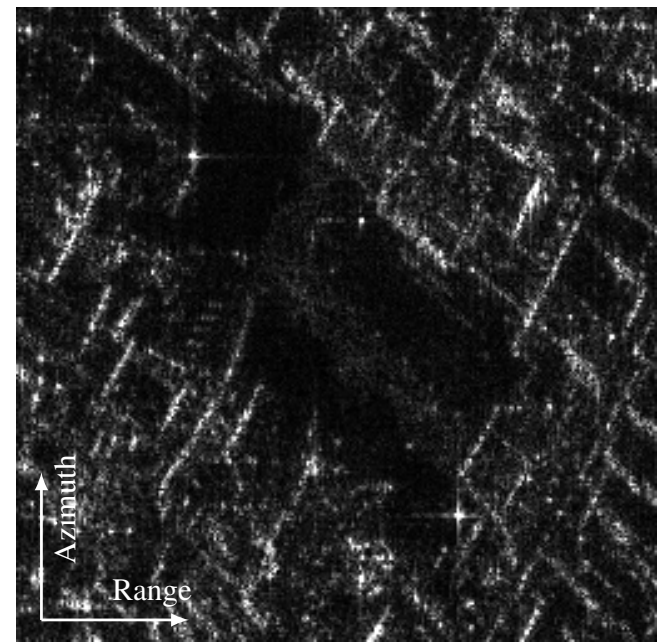

(a) Original image.

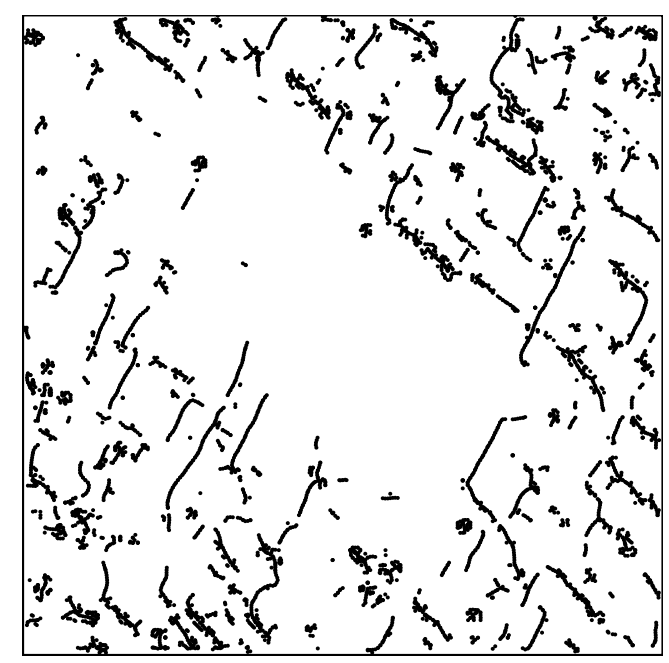

(b) Detected bright line points.

Fig. 3: L'Aquila Piazza del Duomo SAR bright line detection results. Processed from COSMO-SkyMed product (C) Agenzia Spaziale Italiana 2009. All rights reserved.

\section{L'Aquila Piazza del Duomo}

The trained classifier was then used to classify ridge points detected with the same $t_{c}=31 / 3$ in a segment of the SAR image covering the cathedral square (Piazza del Duomo) in the 'old town' area of L'Aquila (Fig. 3a). This area of the image contains very densely-packed buildings and high levels of clutter, with strong overlay and shadow effects in evidence, making manual extraction of bright lines challenging. Fig. $3 b$ shows the bright line points detected using the Bayesian classifier. Note in particular the clarity with which the overlay of the building on the south-east edge of the square has been picked out, and that bright lines have been successfully extracted from high-noise areas (e.g. along the north edge of the square).

\section{Conclusion}

A new approach to the detection of bright lines in very high-resolution single-look urban SAR images is proposed, including the novel application of scale-space ridge detection to SAR data using a single characteristic scale. A classification step based on the naïve Bayes classifier is proposed for selection of the ridge points corresponding to bright lines, and suitable feature variables and corresponding statistical models are suggested. The technique is demonstrated by applying it to a single-look COSMO-SkyMed SAR amplitude image of L'Aquila, Italy are shown.

The problem of distinguishing between bright lines due to direct and double-reflection scattering is currently under study, and more extensive testing is to be carried out both on other SAR images and on other parts of the L'Aquila dataset. Additionally, the potential application of automatic bright line extraction to urban change detection is being explored.

\section{ACKNOWLEDGMENTS}

The authors would like to thank the Remote Sensing Group at the University of Naples Federico II and the Italian Space Agency for providing the COSMO-SkyMed SAR images used in this study, and the UK Engineering and Physical Sciences Research Council (EPSRC) for funding the research project.

\section{REFERENCES}

[1] G. Franceschetti, A. Iodice, and D. Riccio. A canonical problem in electromagnetic backscattering from buildings. Geoscience and Remote Sensing, IEEE Transactions on, 40(8):1787-1801, Aug. 2002.

[2] G. Franceschetti, R. Guida, A. Iodice, D. Riccio, G. Ruello, and U. Stilla Building feature extraction via a deterministic approach: application to real high resolution SAR images. In Geoscience and Remote Sensing Symposium, 2007. IGARSS 2007. IEEE International, pages 2681-2684, July 2007.

[3] R. Guida, A. Iodice, and D. Riccio. Assessment of TerraSAR-X products with a new feature extraction application: Monitoring of cylindrical tanks. Geoscience and Remote Sensing, IEEE Transactions on, 48(2):930-938, Feb. 2010.

[4] R. Guida, A. Iodice, and D. Riccio. Height retrieval of isolated buildings from single high-resolution SAR images. Geoscience and Remote Sensing, IEEE Transactions on, 48(7):2967 -2979, july 2010.

[5] D. Eberly, R. Gardner, B. Morse, S. Pizer, and C. Scharlach. Ridges for image analysis. Journal of Mathematical Imaging and Vision, 4(4):353373, 1994.

[6] T. Lindeberg. Edge detection and ridge detection with automatic scale selection. International Journal of Computer Vision, 30(2):117-154, 1998.

[7] J.Y. Lim and H.S. Stiehl. A generalized discrete scale-space formulation for 2-D and 3-D signals. Scale Space Methods in Computer Vision, pages 132-147, 2003.

[8] David Lewis. Naïve (Bayes) at forty: The independence assumption in information retrieval. In Claire Nédellec and Céline Rouveirol, editors, Machine Learning: ECML-98, volume 1398 of Lecture Notes in Computer Science, pages 4-15. Springer Berlin / Heidelberg, 1998.

[9] A.C. Frery, H.-J. Muller, C.C.F. Yanasse, and S.J.S. Sant'Anna. A model for extremely heterogeneous clutter. Geoscience and Remote Sensing, IEEE Transactions on, 35(3):648-659, May 1997.

[10] C. Tison, J.-M. Nicolas, F. Tupin, and H. Maitre. A new statistical model for Markovian classification of urban areas in high-resolution SAR images. Geoscience and Remote Sensing, IEEE Transactions on, 42(10):2046-2057, Oct. 2004. 